\title{
Constraints on Unparticle Interactions from Particle and Antiparticle Oscillations
}

\author{
Shao-Long Chen ${ }^{1}$, Xiao-Gang He ${ }^{1,2}$, Xue-Qian $\mathrm{Li}^{2}$, Ho-Chin Tsai ${ }^{1}$ and Zheng-Tao Wei ${ }^{2}$ \\ ${ }^{1}$ Department of Physics and Center for Theoretical Sciences, \\ National Taiwan University, Taipei \\ ${ }^{2}$ Department of Physics, Nankai University, Tianjin 300071
}

(Dated: October 28, 2018)

\begin{abstract}
We study unparticle effects on particle and antiparticle osillations in meson-antimeson, and muonium-antimuonium systems. Unlike usual tree level contributions to meson oscillations from heavy particle exchange with small $\Gamma_{12}$, the unparticle may have sizeable contributions to both $M_{12}$ and $\Gamma_{12}$ due to fractional dimension $d_{\mathcal{U}}$ of the unparticle. We find that very stringent constraints on the unparticle and particle interactions can be obtained. If unparticle effect dominates the contributions (which may happen in $D^{0}-\bar{D}^{0}$ mixing) to meson mixing parameters $x$ and $y$, we find that $x / y=\cot \left(\pi d_{\mathcal{U}}\right)$. Interesting constraints on unparticle and particle interactions can also be obtained using muonion and antimuonion oscillation data. We also comment on unparticle effects on CP violation in meson oscillations.
\end{abstract}




\section{INTRODUCTION}

Recently Georgi proposed an interesting idea to describe possible scale invariant effect at low energies by unparticles[1]. Georgi argued that operators $O_{B Z}$ made of BZ fields in the scale invariant sector may interact with operators $O_{S M}$ of dimension $d_{S M}$ made of Standard Model (SM) fields at some high energy scale by exchange particles with large masses, $M_{\mathcal{U}}$, with the generic form $O_{S M} O_{B Z} / M_{\mathcal{U}}^{k}$. At another scale $\Lambda_{\mathcal{U}}$ the BZ sector induce dimensional transmutation, below that scale the BZ operator $O_{B Z}$ matches onto unparticle operator $O_{\mathcal{U}}$ with dimension $d_{\mathcal{U}}$ and the unparticle interaction with SM particles at low energy has the form

$$
\lambda \Lambda_{\mathcal{U}}^{4-d_{S M}-d_{\mathcal{U}}} O_{S M} O_{\mathcal{U}}
$$

Study of unparticle effects has drawn a lot of attentions from collider physics [1, 2], low energy flavor conserving and flavor violating processes [3, 4, 5], long range effects [6], cosmological and astrophysics phenomena [7], to more theoretical studies [8]. In this work we further study unparticle effects on particle and antiparticle oscillations of meson $P$ and antimeson $\bar{P}$, and muonium and antimuonium systems.

Unparticle effects on oscillation in meson and antimeson have been considered previously [4]. Our investigation for meson and antimeson oscillation will focus on some interesting features due to the fractional dimension of unparticle $d_{\mathcal{U}}$. Unlike usual tree level contributions to meson oscillations from heavy particle exchange with small $\Gamma_{12}$, the unparticle may have sizeable contributions to both $M_{12}$ and $\Gamma_{12}$ due to fractional dimension $d_{\mathcal{U}}$ of the unparticle leading to a phase factor $(-1)^{d_{\mathcal{U}}-2}$ in the propagation. If unparticle effect dominates the contributions (which may happen in $D^{0}-\bar{D}^{0}$ mixing) to meson mixing parameters $x$ and $y$, we find that $x / y=\cot \left(\pi d_{\mathcal{U}}\right)$.

Meson-antimeson oscillation exists in several neutral meson systems, $K^{0}-\bar{K}^{0}, D^{0}-\bar{D}^{0}$, $B_{d}^{0}-\bar{B}_{d}^{0}$ and $B_{s}^{0}-\bar{B}_{s}^{0}$. Long distance contributions to oscillation parameters for $K^{0}-\bar{K}^{0}$ are large which causes a large uncertainty in theoretical calculations. We will restrict our calculations for unparticle effects to $D^{0}-\bar{D}^{0}$ and $B_{d, s}^{0}-\bar{B}_{d, s}^{0}$ systems. We find that very stringent constraints on the unparticle and particle interactions can be obtained.

Muonium $(M=(\bar{\mu} e))$ and antimuonium $(\bar{M}=(\bar{e} \mu))$ oscillation may also provide interesting constraints on flavor changing interaction. Experimentally muonium-antimuonium 
oscillation has not been established. Our analysis shows that constraints on unparticle and particle interactions can indeed be obtained using experimental data on muoniumantimuonium oscillation.

\section{MESON AND ANTIMESON OSCILLATIONS}

The mixing of a meson and its antimeson is determined by the off diagonal matrix elements $M_{12}$ and $\Gamma_{12}$ in the Hamiltonian. Their relations to the mass and lifetime differences are given by,

$$
\left(m_{H}-m_{L}\right)-i\left(\Gamma_{H}-\Gamma_{L}\right) / 2=2 \sqrt{\left(M_{12}-i \Gamma_{12} / 2\right)\left(M_{12}^{*}-i \Gamma_{12}^{*} / 2\right)},
$$

where the subscripts "H" and "L" label the mass eigenstates, $\left|P_{H}\right\rangle=p|P\rangle+q|\bar{P}\rangle$ and $\left|P_{L}\right\rangle=p|P\rangle-q|\bar{P}\rangle$, respectively. $p$ and $q$ are normalized as $|p|^{2}+|q|^{2}=1$ and $(q / p)^{2}=$ $\left(M_{12}^{*}-i \Gamma_{12}^{*} / 2\right) /\left(M_{12}-i \Gamma_{12} / 2\right)$. We denote the mass and lifetime differences by $\Delta m=$ $m_{H}-m_{L}$ and $\Delta \Gamma=\Gamma_{H}-\Gamma_{L}$. The parameters $x$ and $y$ are related to $\Delta m$ and $\Delta \Gamma$ by $x=\Delta m / \Gamma$ and $y=\Delta \Gamma / 2 \Gamma$.

There are several possible contributions to $M_{12}$ and $\Gamma_{12}$ from unparticle and particle interactions. The following operators composed of SM fields and derivatives with dimensions less than or equal to 4 invariant under the SM gauge can contribute to meson mixing at tree level,

$$
\begin{array}{ll}
\text { a) : } & \lambda_{Q Q}^{\prime} \Lambda_{\mathcal{U}}^{1-d_{\mathcal{U}}} \bar{Q}_{L} \gamma_{\mu} Q_{L} O_{\mathcal{U}}^{\mu}, \lambda_{U U}^{\prime} \Lambda_{\mathcal{U}}^{1-d_{\mathcal{U}}} \bar{U}_{R} \gamma_{\mu} U_{R} O_{\mathcal{U}}^{\mu}, \lambda_{D D}^{\prime} \Lambda_{\mathcal{U}}^{1-d_{\mathcal{U}}} \bar{D}_{R} \gamma_{\mu} D_{R} O_{\mathcal{U}}^{\mu} \\
b): & i \lambda_{Q Q} \Lambda_{\mathcal{U}}^{-d_{\mathcal{U}}} \bar{Q}_{L} \gamma_{\mu} D^{\mu} Q_{L} O_{\mathcal{U}}, i \lambda_{U U} \Lambda_{\mathcal{U}}^{-d_{\mathcal{U}}} \bar{U}_{R} \gamma_{\mu} D^{\mu} U_{R} O_{\mathcal{U}}, i \lambda_{D D} \Lambda_{\mathcal{U}}^{-d_{\mathcal{U}}} \bar{D}_{R} \gamma_{\mu} D^{\mu} D_{R} O_{\mathcal{U}} \\
c): & i \tilde{\lambda}_{Q Q} \Lambda_{\mathcal{U}}^{-d_{\mathcal{U}}} \bar{Q}_{L} \gamma_{\mu} Q_{L} \partial^{\mu} O_{\mathcal{U}}, i \tilde{\lambda}_{U U} \Lambda_{\mathcal{U}}^{-d_{\mathcal{U}}} \bar{U}_{R} \gamma_{\mu} U_{R} \partial^{\mu} O_{\mathcal{U}}, i \tilde{\lambda}_{D D} \Lambda_{\mathcal{U}}^{-d_{\mathcal{U}}} \bar{D}_{R} \gamma_{\mu} D_{R} \partial^{\mu} O_{\mathcal{U}} ; \\
\text { d) : } & \lambda_{Y U} \Lambda_{\mathcal{U}}^{-d_{\mathcal{U}}} \bar{Q}_{L} H U_{R} O_{\mathcal{U}}, \lambda_{Y D} \Lambda_{\mathcal{U}}^{-d_{\mathcal{U}}} \bar{Q}_{L} \tilde{H}_{R} D_{\mathcal{U}} .
\end{array}
$$

Here $Q_{L}, U_{R}$, and $D_{R}$ are the SM left-handed quark doublet, right-handed up-quark, and right-handed down-quark, respectively.

After using equation of motion for quarks, the interactions in eq.(3) can be parameterized in the following form

For vector $O_{\mathcal{U}}^{\mu}: \mathcal{L}_{V}=\Lambda_{\mathcal{U}}^{1-d_{\mathcal{U}}} \bar{q}_{i}\left(c_{V_{L}}^{i j} \gamma_{\mu} L+c_{V_{R}}^{i j} \gamma_{\mu} R\right) q_{j} O_{\mathcal{U}}^{\mu}$

For scalar $O_{\mathcal{U}}: \mathcal{L}_{S}=\Lambda_{\mathcal{U}}^{1-d_{\mathcal{U}}} \frac{m_{j}}{\Lambda_{\mathcal{U}}} \bar{q}_{i}\left(c_{S_{L}}^{i j} L+c_{S_{R}}^{i j} R\right) q_{j} O_{\mathcal{U}}^{\mu}$ 

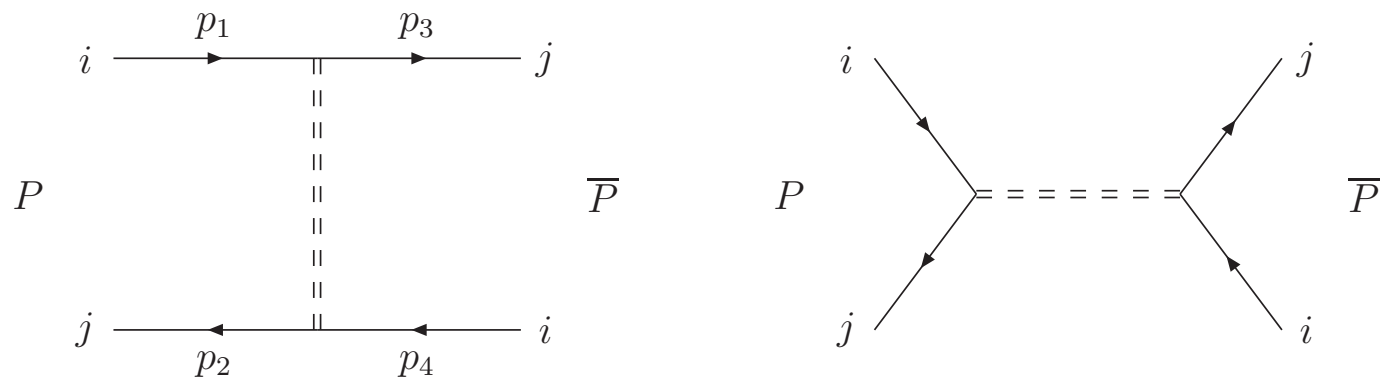

FIG. 1: The $t$ and $s$ channel contributions to meson-antimeson oscillation.

where $q_{i(j)}$ denote quarks with flavor indices $i(j)$. The parameters $c_{V_{L, R}}^{i j}$ and $c_{S_{L, R}}^{i j}$ are different for the interactions listed in a) to d). They are given by

$$
\begin{aligned}
& \text { a): } \quad c_{V_{L}}^{i j}=\lambda_{Q Q}^{\prime i j}, \quad c_{V_{R}}^{i j}=\lambda_{U U, D D}^{\prime i j} ; \\
& b): \quad c_{S_{L}}^{i j}=\frac{1}{m_{j}}\left(\lambda_{Q Q}^{j i *} m_{i}+\lambda_{U U, D D}^{i j} m_{j}\right), \quad c_{S_{R}}^{i j}=\frac{1}{m_{j}}\left(\lambda_{Q Q}^{i j} m_{j}+\lambda_{U U, D D}^{j i *} m_{i}\right) ; \\
& c): \quad c_{S_{L}}^{i j}=\frac{1}{m_{j}}\left(\tilde{\lambda}_{Q Q}^{i j} m_{i}-\tilde{\lambda}_{U U, D D}^{i j} m_{j}\right), \quad c_{S_{R}}^{i j}=\frac{1}{m_{j}}\left(\tilde{\lambda}_{U U, D D}^{i j} m_{i}-\tilde{\lambda}_{Q Q}^{i j} m_{j}\right) ; \\
& \text { d): } \quad c_{S_{L}}^{i j}=v \lambda_{Y F}^{j i *}, \quad c_{S_{R}}^{i j}=v \lambda_{Y F}^{i j} .
\end{aligned}
$$

where $v=\langle H\rangle$ is the vacuum expectation value of $H$. Note that the vector unparticle couplings $c_{V}$ scaled as $\Lambda_{\mathcal{U}}^{1-d_{\mathcal{U}}}$, while scalar unparticle couplings $c_{S}$ scaled as $\Lambda_{\mathcal{U}}^{-d_{\mathcal{U}}}$.

Evaluating the two diagrams in Fig. 1, we obtain

For $O_{\mathcal{U}}^{\mu}$ :

$$
\begin{aligned}
H_{e f f}^{\mathcal{U}} & =-\frac{A_{d_{\mathcal{U}}}}{2 \sin \left(\pi d_{\mathcal{U}}\right)} \Lambda_{\mathcal{U}}^{2\left(1-d_{\mathcal{U}}\right)} e^{-i \pi d_{\mathcal{U}}}\left\{\frac{1}{4}\left(\frac{1}{(s)^{2-d_{\mathcal{U}}}}+\frac{1}{(t)^{2-d_{\mathcal{U}}}}\right)\left(\bar{q}_{i}\left(c_{V_{L}}^{i j} \gamma_{\mu} L+c_{V_{R}}^{i j} \gamma_{\mu} R\right) q_{j}\right)^{2}\right. \\
& \left.+\frac{1}{4}\left(\frac{1}{s(s)^{2-d_{\mathcal{U}}}}+\frac{1}{t(t)^{2-d_{\mathcal{U}}}}\right)\left(\bar{q}_{i}\left(c_{V_{L}}^{i j}\left(m_{i} L-m_{j} R\right)+c_{V_{R}}^{i j}\left(m_{i} R-m_{j} L\right)\right) q_{j}\right)^{2}\right\}
\end{aligned}
$$

For $O_{\mathcal{U}}$ :

$$
H_{\text {eff }}^{\mathcal{U}}=\frac{A_{d_{\mathcal{U}}}}{2 \sin \left(\pi d_{\mathcal{U}}\right)} \Lambda_{\mathcal{U}}^{2\left(1-d_{\mathcal{U}}\right)} \frac{m_{j}^{2}}{\Lambda_{\mathcal{U}}^{2}} e^{-i \pi d_{\mathcal{U}}} \frac{1}{4}\left(\frac{1}{(s)^{2-d_{\mathcal{U}}}}+\frac{1}{(t)^{2-d_{\mathcal{U}}}}\right)\left(\bar{q}_{i}\left(c_{S_{L}}^{i j} L+c_{S_{R}}^{i j} R\right) q_{j}\right)^{2}
$$

Here $A_{d_{\mathcal{U}}}=\left(16 \pi^{5 / 2} /(2 \pi)^{2 d_{\mathcal{U}}}\right) \Gamma\left(d_{\mathcal{U}}+1 / 2\right) /\left(\Gamma\left(d_{\mathcal{U}}-1\right) \Gamma\left(2 d_{\mathcal{U}}\right)\right)$. We have used $\left(i A_{d_{\mathcal{U}}} / 2 \sin \left(\pi d_{\mathcal{U}}\right)\right) \times\left(1 /\left(-p^{2}\right)^{2-d_{\mathcal{U}}}\right)$ and $\left(i A_{d_{\mathcal{U}}} / 2 \sin \left(\pi d_{\mathcal{U}}\right)\right) \times\left(\left(-g^{\mu \nu}+p^{\mu} p^{\nu} / p^{2}\right) /\left(-p^{2}\right)^{2-d_{\mathcal{U}}}\right)$ for scalar and vector unparticle propagators, respectively.

In the systems we are studying, mesons are made of a light (labelled by i) and a heavy quark (labelled by j). In the heavy quark limit, one has $s=t \approx m_{j}^{2} \approx m_{P}^{2}$. With this 
approximation and theoretical matrix elements for the relevant operators, we have

$$
\begin{aligned}
& M_{12}^{\mathcal{U}}=A_{d_{\mathcal{U}}}\left(\frac{m_{P}^{2}}{\Lambda_{\mathcal{U}}^{2}}\right)^{d_{\mathcal{U}}-1} \frac{f_{P}^{2}}{12 m_{P}}\left\{-\left(c_{V}^{i j}\right)^{2}\left(B_{V}-\frac{5}{8} B_{S}\right)-\frac{m_{P}^{2}}{\Lambda_{\mathcal{U}}^{2}}\left(c_{S}^{i j}\right)^{2} \frac{5}{8} B_{S}\right\} \cot \left(d_{\mathcal{U}} \pi\right), \\
& \Gamma_{12}^{\mathcal{U}}=2 M_{12}^{\mathcal{U}} \tan \left(d_{\mathcal{U}} \pi\right) .
\end{aligned}
$$

We have included a missing factor of $1 / 2$ ! due to Wick rotation in previous studies [4]. The parameters $B_{V, S}$ are the bag factors which are equal to 1 in the vacuum saturation and factorization approximation.

We would like to point out some silent features of the unparticle contribution to $M_{12}^{\mathcal{U}}$ and $\Gamma_{12}^{\mathcal{U}}$ due to the phase factor $e^{-i \pi d_{\mathcal{U}}}$. We note that $M_{12}^{\mathcal{U}}$ can have both sign depending on the value of $d_{\mathcal{U}}$ due to the factor $\cot \left(\pi d_{\mathcal{U}}\right)$, therefore if information about the sign can be obtained from other considerations, the dimension $d_{\mathcal{U}}$ can be restricted. There may be a sizeable contribution to $\Gamma_{12}$ at tree level which is not possible for usual tree level heavy particle exchange. For $d_{\mathcal{U}}$ equal to half integers, there is no contribution to $M_{12}$, but there is for $\Gamma_{12}$. Another interesting feature of unparticle contribution is that the ratio $M_{12} /\left(\Gamma_{12} / 2\right)$ of unparticle contribution is related to the unparticle dimension parameter $d_{\mathcal{U}}$ by

$$
\frac{M_{12}^{\mathcal{U}}}{\Gamma_{12}^{\mathcal{U}} / 2}=\cot \left(\pi d_{\mathcal{U}}\right)
$$

If the unparticle contribution dominates meson and antimeson oscillation then the measurements of $M_{12}$ and $\Gamma_{12}$ provide a possible way to determine the dimension parameter $d_{\mathcal{U}}$.

We now present our numerical results on the constraints for unparticle and particle interactions with the assumption of $\mathrm{CP}$ conservation. In this case the unparticle contribution to mass difference $\Delta m^{\mathcal{U}}=2\left|M_{12}^{\mathcal{U}}\right|$ and $x^{\mathcal{U}} / y^{\mathcal{U}}=M_{12}^{\mathcal{U}} /\left(\Gamma_{12}^{\mathcal{U}} / 2\right)=\cot \left(\pi d_{\mathcal{U}}\right)$. We will comment on possible CP violating effects later. The results are shown in Figs. 2] and 3, The constraints are obtained with the vacuum saturation approximation, i.e. $B_{V}=B_{S}=1$ and $f_{D}=0.201 \mathrm{GeV}, f_{B_{d}}=0.216 \mathrm{GeV}$ and $f_{B_{s}}=0.260 \mathrm{GeV}[9]$. $D^{0}-\bar{D}^{0}$ System

Belle and BABAR collaborations have recently published evidence for $D^{0}-\bar{D}^{0}$ oscillation [10]. The Heavy Flavor Averaging Group (HFAG) [11] combined all mixing measurements to obtain world average (WA) values for $x$ and $y$ for CP conserving case with $x=\left(0.87_{-0.34}^{+0.30}\right) \%$ and $y=\left(0.66_{-0.20}^{+0.21}\right) \%$. Short distance contributions in the SM are several 
orders of magnitudes smaller than the central experimental values. There are possible large long distance contributions which are however difficult to have precise predictions. We will assume that the contributions to $x$ and $y$ are purely from unparticle effects. With this assumption, we immediately obtain

$$
\frac{x}{y}=\cot \left(\pi d_{\mathcal{U}}\right) \simeq 1.31 \pm 0.61 .
$$

Although the sign of $x$ and $y$ are both positive, the absolute sign of $M_{12}^{\mathcal{U}}$ cannot be determined from $x$ measurement, therefore $\pi d_{\mathcal{U}}$ can be in the first and third quadrants from the sign of $x / y$. We have

$$
d_{\mathcal{U}}=(0.21+n) \pm 0.07
$$

where $n$ is an integer number which cannot be determined from just information from $x / y$. Note that the experimental errors are still large, consequently the uncertainties of $d_{\mathcal{U}}$ are substantial.

One can also obtain constraints on the couplings $c_{V_{L, R}}$ and $c_{S_{L, R}}$ from $x$ or $y$ for different $d_{\mathcal{U}}$, allowing unparticle contributions to saturate the experimental upper bound on $x_{D}$ or $y_{D}$. As the contributions from unparticle are suppressed by factors of $\left(m_{D}^{2} / \Lambda_{\mathcal{U}}^{2}\right)^{d_{\mathcal{U}}-1}$ and $\left(m_{D}^{2} / \Lambda_{\mathcal{U}}^{2}\right)^{d \mathcal{U}}$ for vector and scalar unparticle respectively, if $n$ is large the contributions are negligible for a fixed $\Lambda_{\mathcal{U}}$. We will therefore just consider the lowest possibilities with phase $\pi d_{\mathcal{U}}$ covering all four quadrants plotting results for $d_{\mathcal{U}}$ in the range of 1 to 3 for illustration in Fig. 2 (solid curves) with $\Lambda_{\mathcal{U}}$ fixed to be $1 \mathrm{TeV}$. In this range, the phase $\pi d_{\mathcal{U}}$ will cover all four quadrants. At $d_{\mathcal{U}}$ equal to half integers $(1.5,2.5)$, there is no contribution to $\Delta m^{\mathcal{U}}$, and therefore there are no constraints on $c_{V, S}$. This is indicated by the two peaks at $d_{\mathcal{U}}=1.5$ and 2.5 in Fig. 2. At $d_{\mathcal{U}}$ equal to $1, \sin \left(\pi d_{\mathcal{U}}\right)=0$, naively the contribution blows off. However, at $d_{\mathcal{U}}=1, A_{d_{\mathcal{U}}} / \sin \left(\pi d_{\mathcal{U}}\right)$ is finite and therefore there is finite contribution as should be for a dimension one particle. For other integers, the contribution blows off. For these reasons, when reading Fig. 2, one should not taken values too close to integers larger than 1 and half integers for $d_{\mathcal{U}}$. One can also use data on $y_{D}$ to constrain $c_{V, S}$. The results are shown in Fig. 3 (solid curve). Note that in this case, at $d_{\mathcal{U}}=1$, there is no contribution to $y_{D}$ because $A_{d_{\mathcal{U}}}=0$.

From Figs. 2 and 3 (solid curves), we see that constraints from $x_{D}$ and $y_{D}$ give similar constraints for $c_{V, S}$ when away from integers and half-integers. The constraint for $c_{S}$ is 
weaker than $c_{V}$ because of the relative suppression factor $m_{D}^{2} / \Lambda_{\mathcal{U}}^{2}$ as pointed out earlier. Smaller $d_{\mathcal{U}}$ give stronger constraints on $c_{V, S}$.

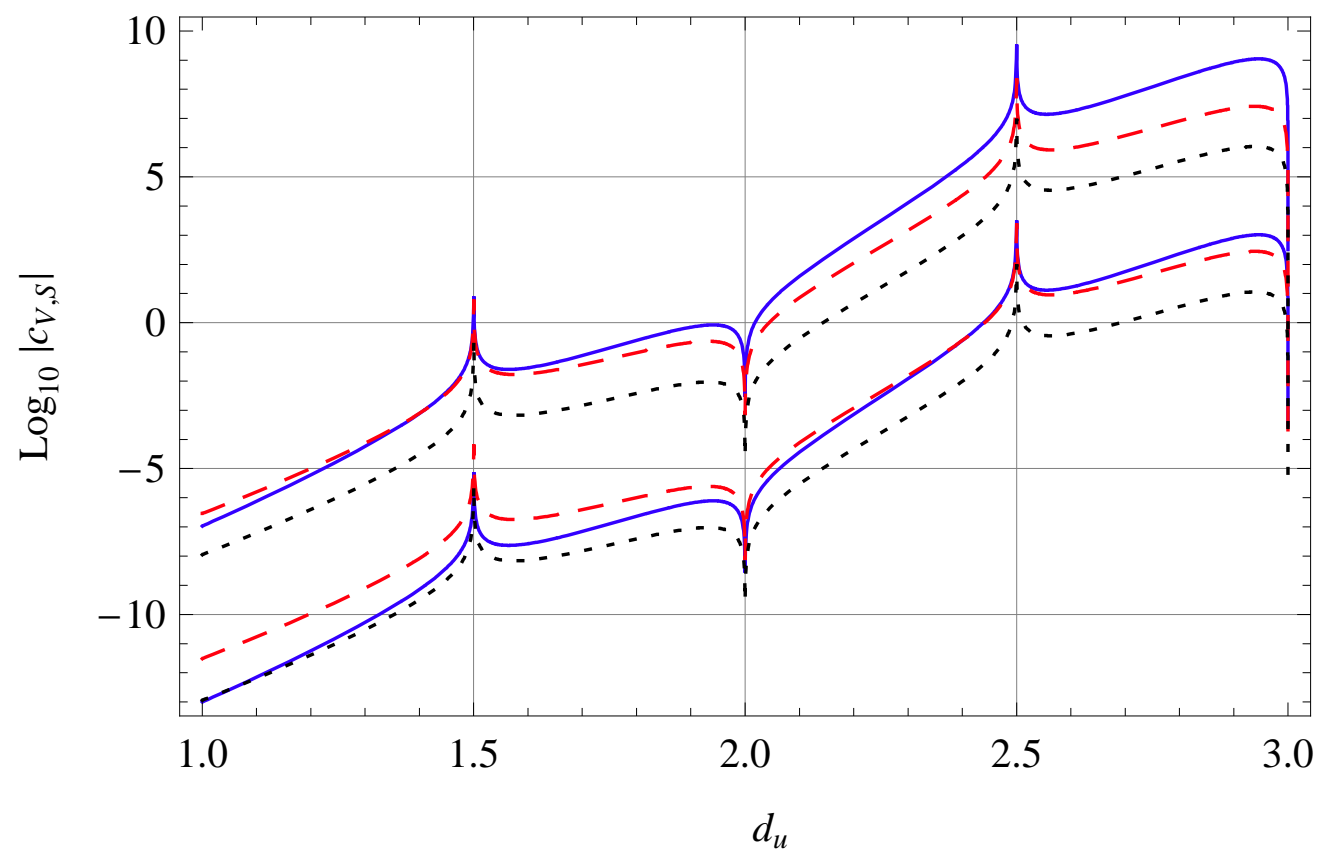

FIG. 2: Constraints on the coupling $c_{S}$ (upper curves) and $c_{V}$ (lower curves) as functions of $d_{\mathcal{U}}$ from meson-antimeson oscillation with $\Lambda_{\mathcal{U}}=1 \mathrm{TeV}$ by fitting the mass differences $\Delta m$. The solid (blue), dashed (red), and dotted (black) curves are for $D^{0}-\bar{D}^{0}, B_{d}-\bar{B}_{d}$ and $B_{s}-\bar{B}_{s}$, respectively.

$B_{d}^{0}-\bar{B}_{d}^{0}$ System

$\Delta m_{B_{d}}$ has been measured to be $(0.507 \pm 0.005) \mathrm{ps}^{-1}(x=0.776 \pm 0.008)[12$, 13]. A non-zero $\Delta \Gamma_{B_{d}}(y)$ has not been established. The SM prediction for $\Delta m_{B_{d}}$ agrees with data very well. The prediction for $\Delta \Gamma_{B_{d}}$ is $-\left(26.7_{-6.5}^{+5.8}\right) \times 10^{-4} \mathrm{ps}^{-1}$ [14] which is very small to be measured experimentally. Since SM prediction for $\Delta m_{B_{d}}$ agrees with data, in our analysis to obtain constraints on the couplings $c_{V, S}$ we will just allow the unparticle contributions to vary within $2 \sigma$ of experimental error bar.

Since the SM prediction for $M_{12}^{S M}$ is positive, if the unparticle contribution is required to increase (decrease) the value for $\Delta m_{B_{d}}$ relative to the SM value, then $\pi d_{\mathcal{U}}$ needs to be in second and fourth (first and third) quadrants. In Fig. 2, we show the results for constraints on $c_{V, S}$ assuming the unparticle contributions saturate $2 \sigma$ of experimental error bar for $\Delta m_{B_{d}}$. The constraints are similar to those obtained from $D^{0}-\bar{D}^{0}$ oscillation. The 


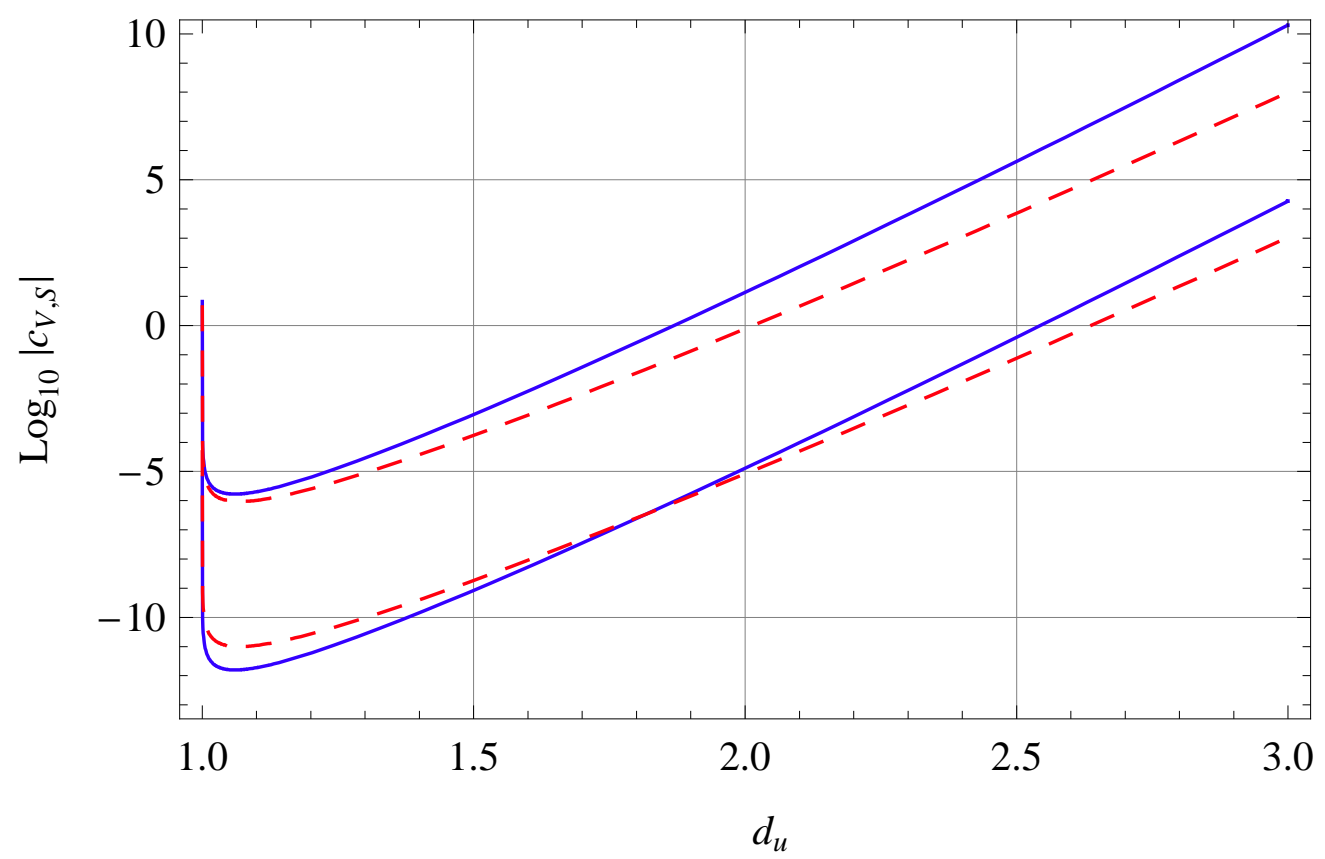

FIG. 3: Constraints on the coupling $c_{S}$ (upper curves) and $c_{V}$ (lower curves) from meson-antimeson oscillation with $\Lambda_{\mathcal{U}}=1 \mathrm{TeV}$ by fitting the width differences $\Delta \Gamma$. The solid (blue) and dashed (red) curves are for $D^{0}-\bar{D}^{0}$ and $B_{s}-\bar{B}_{s}$, respectively.

predicted value for $y_{B_{d}}^{\mathcal{U}}$ is given by $y_{B_{d}}^{\mathcal{U}}=x_{B_{d}}^{\mathcal{U}} \tan \left(\pi d_{\mathcal{U}}\right)$ which can be as large as present experimental upper bound since at $d_{\mathcal{U}}$ close to half integers $\cot \left(\pi d_{\mathcal{U}}\right)$ can be very large, and at half integers there is no constraint from $\Delta m$. Future experiments may tell us more. $B_{s}^{0}-\bar{B}_{s}^{0}$ System

For $B_{s}^{0}-\bar{B}_{s}^{0}, \Delta m_{B_{c}}$ is measured to be $(17.77 \pm 0.12) \mathrm{ps}^{-1}$ [15], and $\Delta \Gamma_{B_{s}}=$ $-\left(0.084_{-0.050}^{+0.055}\right) \mathrm{ps}^{-1}[12,13]$. SM best fits are $\Delta m_{B_{s}}=(19.3 \pm 6.68) \mathrm{ps}^{-1}$ and $\Delta \Gamma_{B_{s}}=$ $-(0.096 \pm 0.039) \mathrm{ps}^{-1}[14]$. There are differences for central values of SM predictions and experimental measurements. Taking these central values and attributing the differences are due to unparticle effects, one would favor $\pi d_{\mathcal{U}}$ to be in the first or third quadrants. Since both SM predictions and experimental measurements have large errors and they agree with in error bars, we will present our constraints on $c_{V, S}$ taking, again, $2 \sigma$ experimental error bars for both $\Delta m$ and $\Delta \Gamma$. The results are shown in Figs. 2 and 3 . The constraints on $c_{V, S}$ are similar to those obtained from $D^{0}-\bar{D}^{0}$ oscillation. 


\section{MUONIUM-ANTIMUONIUM OSCILLATION}

For muonium and antimuonium oscillation to occur, there must be flavor changing interactions. To the lowest order, the following unparticle and particle interaction operators will contribute,

$$
\begin{array}{ll}
\text { a) : } & \lambda_{L L}^{\prime} \Lambda_{\mathcal{U}}^{1-d_{\mathcal{U}}} \bar{L}_{L} \gamma_{\mu} L_{L} O_{\mathcal{U}}^{\mu}, \lambda_{E E}^{\prime} \Lambda_{\mathcal{U}}^{1-d_{\mathcal{U}}} \bar{E}_{R} \gamma_{\mu} E_{R} O_{\mathcal{U}}^{\mu} \\
\text { b) : } & i \lambda_{L L} \Lambda_{\mathcal{U}}^{-d_{\mathcal{U}}} \bar{L}_{L} \gamma_{\mu} D^{\mu} L_{L} O_{\mathcal{U}}, i \lambda_{E E} \Lambda_{\mathcal{U}}^{-d_{\mathcal{U}}} \bar{E}_{R} \gamma_{\mu} D^{\mu} E_{R} O_{\mathcal{U}} \\
c): & i \tilde{\lambda}_{L L} \Lambda_{\mathcal{U}}^{-d_{\mathcal{U}}} \bar{L}_{L} \gamma_{\mu} L_{L} \partial^{\mu} O_{\mathcal{U}}, i \tilde{\lambda}_{E E} \Lambda_{\mathcal{U}}^{-d_{\mathcal{U}}} \bar{E}_{R} \gamma_{\mu} E_{R} \partial^{\mu} O_{\mathcal{U}} \\
\text { d) : } & \lambda_{Y E} \Lambda_{\mathcal{U}}^{-d_{\mathcal{U}}} \bar{L}_{L} \tilde{H} E_{R} O_{\mathcal{U}} .
\end{array}
$$

At tree level, exchange of unparticles will generate $\bar{\mu} \Gamma_{1} e \bar{\mu} \Gamma_{2} e$ type of matrix elements. The operators has the same form given in eq.(6) with appropriate replacements of quarks by letptons and the associated couplings.

The SM prediction for muonium and antimuonium oscillation is extremely small. Observation of this oscillation at a substantially larger rate will be an indication of new physics. Experimentally, no oscillation has been observed. The current upper limit for the probability of spontaneous muonium to antimuonium conversion was established at $P_{\bar{M} M} \leq 8.3 \times 10^{-11}$ (90\% C.L.) in 0.1 T magnetic field [16].

In the absence of external electromagnetic fields, the probability $P_{\bar{M} M}$ of observing a transition can be written as [17] $P_{\bar{M} M}(0 \mathrm{~T}) \simeq|\delta|^{2} /\left(2 \Gamma_{\mu}^{2}\right)$, where $\delta \equiv 2\left\langle\bar{M}\left|H_{\text {eff }}\right| M\right\rangle$ and $\Gamma_{\mu}$ is the muon decay width. Here the effective Hamiltonian is defined as $H_{e f f}=$ $\left(G_{\bar{M} M} / \sqrt{2}\right) \bar{\mu} \Gamma_{1} e \bar{\mu} \Gamma_{2} e$. For the $\Gamma_{1} \times \Gamma_{2}=(V \pm A)^{2}$ type Hamiltonian $\left(\bar{\mu} \gamma_{\lambda}\left(1-\gamma_{5}\right) e\right)^{2}$, the transition amplitude is given by $\delta=16 G_{\bar{M} M} /\left(\sqrt{2} \pi a^{3}\right)$ for both triplet and singlet muonium states, where $a \simeq\left(\alpha m_{e}\right)^{-1}$ is the Bohr radius. But for $(S \pm P)^{2}$ type we have $\delta=-4 G_{\bar{M} M} /\left(\sqrt{2} \pi a^{3}\right)$ for both triplet and singlet muonium [18].

As for our case, omitting $m_{e}$, the contributions corresponding to parameters $c_{V_{L, R}}$ are $(V \pm A)^{2}+(S \pm P)^{2}$ type and to parameters $c_{S_{L, R}}$ are $(S \pm P)^{2}$ type. Therefore we have $\delta$ given by $\delta=12 G_{\bar{M} M} /\left(\sqrt{2} \pi a^{3}\right)$ and $\delta=-4 G_{\bar{M} M} /\left(\sqrt{2} \pi a^{3}\right)$ for $c_{V_{L, R}}$ and $c_{S_{L, R}}$ respectively.

It is important to note that the probability $P_{\bar{M} M}$ has strong magnetic field dependence which usually occurs in experimental situation. With an external magnetic field, there is a reduction factor $S_{B}$, i.e. $P_{\bar{M} M}(B)=S_{B} P_{\bar{M} M}(0 \mathrm{~T})$. The magnetic field correction factor $S_{B}$ describes the suppression of the conversion in the external magnetic field due to the removal 
of degeneracy between corresponding levels in $\bar{M}$ and $M$. One has $S_{B}=0.35$ for $(V \pm A)^{2}$ and $(S \pm P)^{2}$ type interactions at $B=0.1 \mathrm{~T}[16,19]$. Using this experimental information, one obtains the usual constraint $G_{M \bar{M}}<3.0 \times 10^{-3} G_{F}$ for $(V \pm A)^{2}$ type interaction [16]. Applying to our case we can put constraints on the relevant parameters and obtain

$$
\begin{aligned}
& \left|\frac{A_{d_{\mathcal{U}}}}{16 \sin \left(d_{\mathcal{U}} \pi\right) m_{M}^{2}}\left(\frac{m_{M}^{2}}{\Lambda_{\mathcal{U}}^{2}}\right)^{d_{\mathcal{U}}-1}\left(c_{V_{L}, R}^{\mu e}\right)^{2}\right| \leq \frac{4.0 \times 10^{-3} G_{F}}{\sqrt{2}}, \\
& \left|\frac{A_{d_{\mathcal{U}}}}{16 \sin \left(d_{\mathcal{U}} \pi\right) m_{M}^{2}}\left(\frac{m_{M}^{2}}{\Lambda_{\mathcal{U}}^{2}}\right)^{d_{\mathcal{U}}}\left(c_{S_{L, R}}^{\mu e}\right)^{2}\right| \leq \frac{1.2 \times 10^{-2} G_{F}}{\sqrt{2}} .
\end{aligned}
$$

where $G_{F}$ is the Fermi constant.

Using eq.(12), one can obtain constraints on $c_{V, S}$ for given $\Lambda_{\mathcal{U}}$ and $d_{\mathcal{U}}$. The constraints for $c_{V, S}$ are shown in Fig 4 for $\Lambda_{\mathcal{U}}=1 \mathrm{TeV}$. At $d_{\mathcal{U}}$ equal to integers larger than 1 the contribution to $\delta$ blows off due to the appearance of $\sin \left(\pi d_{\mathcal{U}}\right)$ in the denominator of eq.(6) and therefore one should take values away from $d_{\mathcal{U}}$ close to integers. We see that stringent constraint can be obtained on $c_{V}$ for small $d_{\mathcal{U}}$. The constraint for $c_{S}$ is weak because the suppression factor of $m_{\mu}^{2} / \Lambda_{\mathcal{U}}^{2}$ compared with that for $c_{V}$. In general the constraints are weaker compared with those obtained from meson and antimeson oscillations since the suppression factors are now $\left(m_{\mu}^{2} / \Lambda_{\mathcal{U}}^{2}\right)^{d_{\mathcal{U}}-1}$ and $\left(m_{\mu}^{2} / \Lambda_{\mathcal{U}}^{2}\right)^{d_{\mathcal{U}}}$ for vector and scalar unparticle contributions which are more severe than that for meson-antimeson oscillation cases.

\section{DISCUSSIONS AND CONCLUSIONS}

In our previous discussions, we have assumed that there is no $\mathrm{CP}$ violation in the interactions between unparticles and particles. We now briefly comment on some implications for CP violation. If the parameters $\lambda, \tilde{\lambda}$ and $\lambda_{Y}$ are complex, CP is violated. There may be chance to have large enough CP asymmetry for $A_{S L}=\left(1-|q / p|^{4}\right) /\left(1+|q / p|^{4}\right)$ which may be observed by measuring meson and antimeson semi-leptonic decays. Let us take $B_{d}-\bar{B}_{d}$ mixing for discussions since there are a large number of $B_{d}$ mesons produced at B-factories at KEK and SLAC and more detailed study could be carried out in the near future.

In the SM, $A_{S L}$ for $B_{d}-\bar{B}_{d}$ system is predicted to be very small $\left(<10^{-3}\right)$. The reasons for this are two folds: small $\Gamma_{12}$ and small relative $\mathrm{CP}$ violating phase between $M_{12}$ ad $\Gamma_{12}$. With unparticle interactions, $\Gamma_{12}$ can be much larger than the SM prediction as shown

before, and with non-zero $\mathrm{CP}$ violating phases for $\lambda, \tilde{\lambda}$ and $\lambda_{Y}$ relative $\mathrm{CP}$ violating phase 


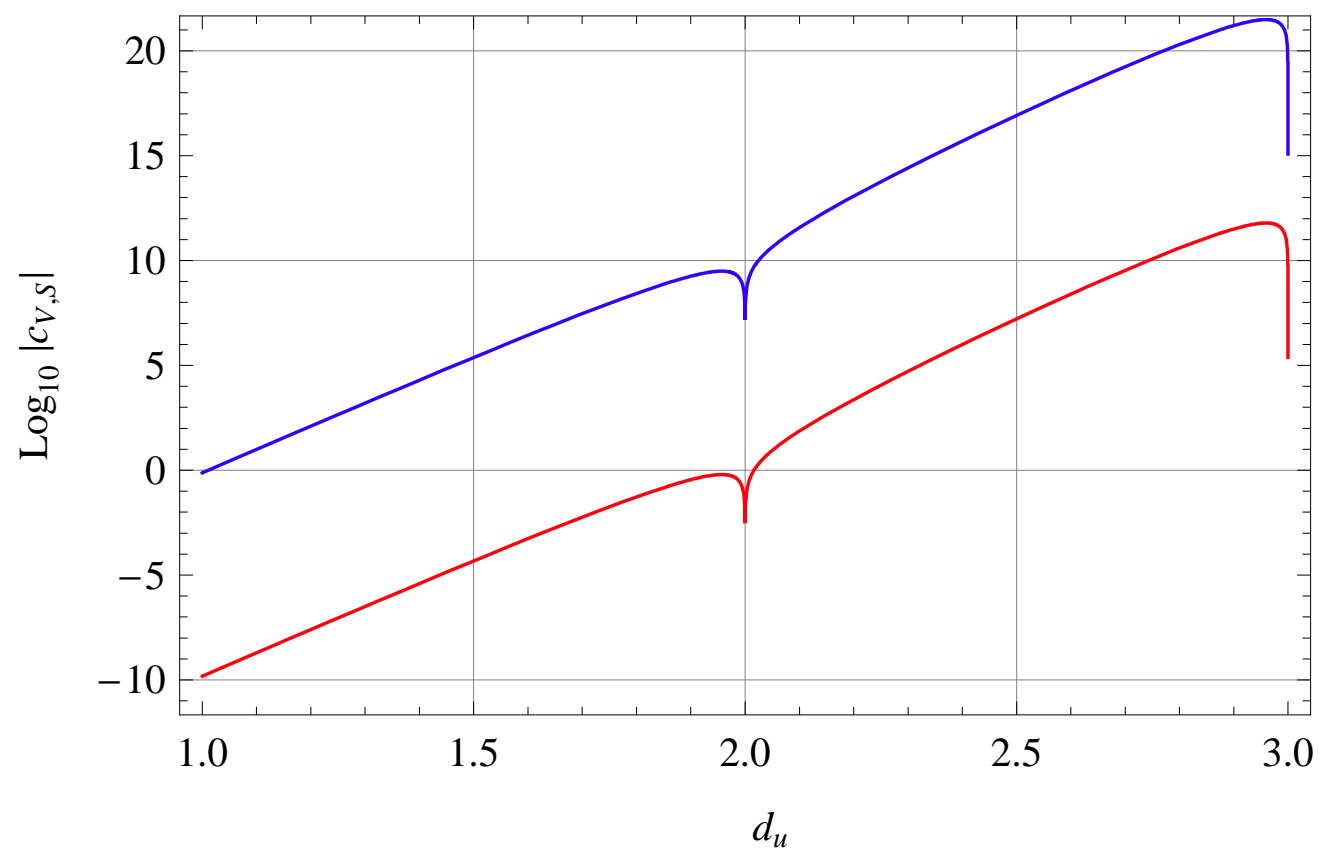

FIG. 4: Constraints on the coupling $c_{V, S}$ from muonium-antimuonium oscillation with $\Lambda_{\mathcal{U}}=1$ $\mathrm{TeV}$. The upper and lower curves are for $c_{S}$ and $c_{V}$ respectively.

between $\Delta m_{12}$ and $\Gamma_{12}$ can be generated. It is possible to have a sizeable $A_{S L}$. To a good approximation, we have, $A_{S L} \approx\left|\Gamma_{12}^{\mathcal{U}}\right| \sin \phi /\left|M_{12}^{\text {total }}\right|$. Here $\phi$ is a relative $\mathrm{CP}$ violating phase between total $M_{12}^{\text {total }}$ and $\Gamma_{12}^{\mathcal{U}}$ which is unknown.

Experimentally, $A_{S L}$ is constrained to be [12] $-0.0049 \pm 0.0038$. Allowing the unparticle contribution to saturate experimental upper bound on $y_{B_{d}}, A_{S L}$ can easily reach present constraint. Similar situation occurs for CP violation in $B_{s}-\bar{B}_{s}$ system. Measurements of $A_{S L}$ can also provide information about unparticle interactions.

To summarize, we have studied unparticle effects on particle and antiparticle oscillations in meson-antimeson, and muonium-antimuonium systems. We found that unlike usual tree level contributions to meson oscillations from heavy particle exchange with small $\Gamma_{12}$, the unparticle may have sizeable contributions to both $M_{12}$ and $\Gamma_{12}$ due to the fractional dimension $d_{\mathcal{U}}$ of the unparticle. Numerically we found that very stringent constraints on the unparticle and particle interactions can be obtained. If unparticle effect dominates the contributions (which may happen in $D^{0}-\bar{D}^{0}$ mixing) to meson mixing parameters $x$ and $y$, $x / y=\cot \left(\pi d_{\mathcal{U}}\right)$. New constraints on unparticle and particle interactions can also be ob- 
tained using muonium and antimuonium oscillation data. Unparticle interactions can also induce large $\mathrm{CP}$ violation in meson oscillations.

Acknowledgments The work of authors was supported in part by the NSC, NCTS and NNSF.

[1] H. Georgi, Phys. Rev. Lett. 98, 221601 (2007) arXiv:hep-ph/0703260; H. Georgi, Phys. Lett. B 650, 275 (2007) arXiv:0704.2457 [hep-ph]].

[2] K. Cheung, W. Y. Keung and T. C. Yuan, Phys. Rev. Lett. 99, 051803 (2007) arXiv:0704.2588 [hep-ph]]; P. J. Fox, A. Rajaraman and Y. Shirman, arXiv:0705.3092 [hep-ph]; N. Greiner, Phys. Lett. B 653, 75 (2007) arXiv:0705.3518 [hep-ph]]; S. L. Chen and X. G. He, arXiv:0705.3946 [hep-ph]; P. Mathews and V. Ravindran, arXiv:0705.4599 [hep-ph]. M. Bander, J. L. Feng, A. Rajaraman and Y. Shirman, arXiv:0706.2677 [hep-ph]; T. G. Rizzo, arXiv:0706.3025 [hep-ph]; K. Cheung, W. Y. Keung and T. C. Yuan, Phys. Rev. D 76, 055003 (2007) arXiv:0706.3155 [hep-ph]]; T. Kikuchi and N. Okada, arXiv:0707.0893 [hepph]; D. Choudhury and D. K. Ghosh, arXiv:0707.2074 [hep-ph]; H. Zhang, C. S. Li and Z. Li, arXiv:0707.2132 [hep-ph]; N. G. Deshpande, X. G. He and J. Jiang, arXiv:0707.2959 [hep-ph]; A. Delgado, J. R. Espinosa and M. Quiros, arXiv:0707.4309 [hep-ph]. M. x. Luo, W. Wu and G. h. Zhu, arXiv:0708.0671 [hep-ph]; A. T. Alan and N. K. Pak, arXiv:0708.3802 [hep-ph]; T. i. Hur, P. Ko and X. H. Wu, arXiv:0709.0629 [hep-ph]; S. Majhi, arXiv:0709.1960 [hepph]; M. C. Kumar, P. Mathews, V. Ravindran and A. Tripathi, arXiv:0709.2478 [hep-ph]; K. m. Cheung, W. Y. Keung and T. C. Yuan, arXiv:0710.2230 [hep-ph].

[3] G. J. Ding and M. L. Yan, arXiv:0705.0794 [hep-ph]; Y. Liao, Phys. Rev. D 76, 056006 (2007) arXiv:0705.0837 [hep-ph]]; S. Zhou, arXiv:0706.0302 [hep-ph]; G. J. Ding and M. L. Yan, arXiv:0706.0325 [hep-ph]; S. L. Chen, X. G. He and H. C. Tsai, arXiv:0707.0187 [hep-ph]; R. Zwicky, arXiv:0707.0677 [hep-ph]; X. Q. Li, Y. Liu and Z. T. Wei, arXiv:0707.2285 [hepph]; G. Bhattacharyya, D. Choudhury and D. K. Ghosh, arXiv:0708.2835 [hep-ph]. A. B. Balantekin and K. O. Ozansoy, arXiv:0710.0028 [hep-ph]; E. O. Iltan, arXiv:0710.2677 [hep-ph].

[4] M. Luo and G. Zhu, arXiv:0704.3532 [hep-ph]; C. H. Chen and C. Q. Geng, arXiv:0705.0689 
[hep-ph]; X. Q. Li and Z. T. Wei, Phys. Lett. B 651, 380 (2007) arXiv:0705.1821 [hep-ph]]. R. Mohanta and A. K. Giri, arXiv:0707.1234 [hep-ph]; A. Lenz, Phys. Rev. D 76, 065006 (2007) arXiv:0707.1535 [hep-ph]].

[5] T. M. Aliev, A. S. Cornell and N. Gaur, arXiv:0705.1326 [hep-ph]; C. D. Lu, W. Wang and Y. M. Wang, Phys. Rev. D 76, 077701 (2007) arXiv:0705.2909 [hep-ph]]; D. Choudhury, D. K. Ghosh and Mamta, arXiv:0705.3637 [hep-ph]; T. M. Aliev, A. S. Cornell and N. Gaur, JHEP 0707, 072 (2007) arXiv:0705.4542 [hep-ph]]; C. H. Chen and C. Q. Geng, Phys. Rev. D 76, 036007 (2007) arXiv:0706.0850 [hep-ph]]; C. S. Huang and X. H. Wu, arXiv:0707.1268 [hep-ph]; R. Mohanta and A. K. Giri, Phys. Rev. D 76, 057701 (2007) arXiv:0707.3308 [hepph]]; C. H. Chen and C. Q. Geng, arXiv:0709.0235 [hep-ph]; G. j. Ding and M. L. Yan, arXiv:0709.3435 [hep-ph]; T. M. Aliev and M. Savci, arXiv:0710.1505 [hep-ph].

[6] Y. Liao and J. Y. Liu, arXiv:0706.1284 [hep-ph]; H. Goldberg and P. Nath, arXiv:0706.3898 [hep-ph]; N. G. Deshpande, S. D. H. Hsu and J. Jiang, arXiv:0708.2735 [hep-ph]; S. Das, S. Mohanty and K. Rao, arXiv:0709.2583 [hep-ph].

[7] H. Davoudiasl, arXiv:0705.3636 [hep-ph]; S. Hannestad, G. Raffelt and Y. Y. Y. Wong, arXiv:0708.1404 [hep-ph]; P. K. Das, arXiv:0708.2812 [hep-ph]; D. Majumdar, arXiv:0708.3485 [hep-ph]; A. Freitas and D. Wyler, arXiv:0708.4339 [hep-ph]; L. Anchordoqui and H. Goldberg, arXiv:0709.0678 [hep-ph]; J. McDonald, arXiv:0709.2350 [hep-ph].

[8] M. A. Stephanov, Phys. Rev. D 76, 035008 (2007) arXiv:0705.3049 [hep-ph]]. Y. Nakayama, arXiv:0707.2451 [hep-ph]; T. A. Ryttov and F. Sannino, arXiv:0707.3166 [hep-th]; M. Neubert, arXiv:0708.0036 [hep-ph]; Y. Liao, arXiv:0708.3327 [hep-ph]; I. Gogoladze, N. Okada and Q. Shafi, arXiv:0708.4405 [hep-ph].

[9] M. Okamoto, PoS LAT2005, 013 (2006) arXiv:hep-lat/0510113.

[10] M. Staric et al. [Belle Collaboration], Phys. Rev. Lett. 98, 211803 (2007) arXiv:hep-ex/0703036]; K. Abe et al. [BELLE Collaboration], arXiv:0704.1000 [hepex]. B. Aubert et al. [BABAR Collaboration], Phys. Rev. Lett. 98, 211802 (2007) arXiv:hep-ex/0703020.

[11] Heavy Flavour Average Group (HFAG), http://www.slac.stanford.edu/xorg/hfag/charm/index.html

[12] W. M. Yao et al. [Particle Data Group], J. Phys. G 33, 1 (2006);

[13] Heavy Flavour Average Group (HFAG) for the 2007 web update of the Particle Data Group review, http://www.slac.stanford.edu/xorg/hfag/osc/PDG_2007/\#DG 
[14] A. Lenz and U. Nierste, JHEP 06 (2007) 072 [arXiv:hep-ph/0612167].

[15] A. Abulencia et al. [CDF Collaboration], arXiv:hep-ex/0609040; A. Abulencia [CDF - Run II Collaboration], Phys. Rev. Lett. 97 (2006) 062003 arXiv:hep-ex/0606027; V. M. Abazov et al. [D0 Collaboration], Phys. Rev. Lett. 97 (2006) 021802 arXiv:hep-ex/0603029].

[16] L. Willmann et al., Phys. Rev. Lett. 82, 49 (1999) arXiv:9807011[hep-ex].

[17] G. Feinberg and S. Weinberg, Phys. Rev. Lett. 6, 381 (1961). Phys. Rev. 123, 1439 (1961). M. L. Swartz, Phys. Rev. D 40, 1521 (1989).

[18] W. S. Hou and G. G. Wong, Phys. Rev. D 53, 1537 (1996) arXiv:hep-ph/9504311. V. Pleitez, Phys. Rev. D 61, 057903 (2000) [arXiv:hep-ph/9905406].

[19] K. Horikawa and K. Sasaki, Phys. Rev. D 53, 560 (1996) arXiv:hep-ph/9504218. 